\title{
Evaluación del Método de Aprendizaje Basado en la Investigación (MABI) en el CICS UMA-IPN
}

\section{Evaluation of the Inquiry-Based Learning Method (MABI) at CICS UMA-IPN}

DOI: $10.46932 / \mathrm{sfjdv2n5-055}$

Received in: Oct 1st, 2021

Accepted in: Dec 30th, 2021

\author{
Claudio Francisco Hernández Rodríguez \\ $\mathrm{M}$ en $\mathrm{C}$ \\ Instituto Politécnico Nacional \\ E-mail: chilapense@gmail.com \\ Blanca Elisa Pérez Magaña \\ $\mathrm{M}$ en $\mathrm{C}$ \\ Instituto Politécnico Nacional \\ E-mail: blancaelisa1@ hotmail.com \\ Carlos Quiroz Téllez \\ M C B \\ Instituto Politécnico Nacional \\ E-mail: cquirozte@ipn.mx
}

\section{RESUMEN}

El método basado en la investigación (MABI), emplea la Participación Referenciada y distintas técnicas de aprendizaje en el aula, apoyadas por las distintas herramientas didácticas, métodos educativos y los sistemas de información y comunicación, TIC'S. El objetivo de esta estrategia es mejorar y flexibilizar el Método de Aprendizaje Basado en la Investigación (MABI) a fin de que, no perdiendo su esencia, se adapte a las condiciones del docente y de los alumnos de los distintos currículos, a fin de elevar el nivel de aprendizaje de los alumnos. Este método se describe en la planeación didáctica del Programa académico: Médico Cirujano y Partero la Unidad de Aprendizaje: Ap. Cardiovascular y Sistema Hematopoyético, (Bioquímica) utilizado en la 41 Generación. Los resultados obtenidos fueron que el índice de reprobación de los alumnos disminuyo. Ya que aproximadamente el número de alumnos reprobados eran entre 10 o 12 alumnos por grupo en las generaciones pasadas en que solo se aplicaba un examen único y ahora después de aplicar este método el índice de reprobación es entre 2 y 4 alumnos reprobados por grupo de un total de 40 alumnos, obteniendo muy buenos resultados.

Palabras clave: Evaluación, Aprendizaje, MABI.

\section{ABSTRACT}

Method Based Learning Research (MABI), Referenced method employs different techniques and learning in the classroom participation, supported by various teaching tools, educational methods and information and communication systems, TIC'S. The aim of this strategy is to improve and streamline Method Based Learning Research (MABI) so that, not losing its essence, it suits the conditions of teachers and students of different curricula, in order to raise the level of student learning. This method is described in educational planning the academic program: Surgeon and Obstetrician Learning Unit. Ap Cardiovascular and 
Hematopoietic System (Biochemistry) used in the 41 generation. The results were that the failure rate of students decreased. Since about the number of passing students were between 10 to 12 students per group in past generations that only a single exam and now after applying this method, the failure rate is between 2 and 4 students reproved by group is applied a total of 40 students, with very good results.

Key words: Evaluation, Learning, MABI

\section{INTRODUCCIÓN}

A partir del año 2001 el Instituto Politécnico Nacional inicia un gran proyecto a través de la estrategia "Diseñemos el futuro" 6, que promueve la renovación institucional, responder con mayor calidad y pertinencia a las necesidades de su comunidad y del país. Como resultado de estas necesidades se crea el Método de Aprendizaje Basado en la Investigación que tiene como instrumento natural el uso de las TIC y los alumnos utilizan la web como instrumento de comunicación e información para sus actividades El aprendizaje es un proceso corresponsable, participativo y permanente, donde intervienen: el aprendiz, docente, condiscípulos, institución y demás factores sociales donde se desenvuelve el estudiante. Como elemento del aprendizaje está la investigación, que se realiza a partir de hechos reales que son abordados sobre la base del desarrollo científico. Los resultados de la investigación se materializan en marcos conceptuales que sustentan el marco teórico del aprendizaje y generan la respuesta social, mediante prácticas que permiten su transformación, favoreciendo así el saber hacer. Como elementos facilitadores del aprendizaje están las tecnologías educativas y las (TIC) tecnología de información y comunicación, que permiten una acción de enseñanza aprendizaje más allá de las paredes de las aulas.

\section{MARCO TEÓRICO}

El aprendizaje es un proceso corresponsable, participativo y permanente, donde intervienen: el aprendiz, docente, condiscípulos, institución y demás factores sociales donde se desenvuelve el estudiante. Como elemento detonador del aprendizaje está la investigación, que se realiza a partir de hechos reales que son abordados sobre la base del desarrollo científico del estado del arte. Los resultados de la investigación se materializan en marcos conceptuales que sustentan el marco teórico del aprendizaje y generan la respuesta social, mediante prácticas que permiten su transformación, favoreciendo así el saber hacer. Como elementos facilitadores del aprendizaje están las tecnologías educativas y las (TIC) tecnología de información y comunicación, que permiten una acción de enseñanza aprendizaje más allá de las paredes de las aulas. (Gregorio, 2007).

(Light G. y Roy C. 2009) menciona : "Los maestros frecuentemente ven el aprendizaje como un resultado en términos de un "estado de conocimiento" que alcanzan los alumnos como individuos aislados más que como un resultado en términos de un proceso de construcción que alcanzan dentro de una situación social integradora". 
También refiere una situación que es una cuestión de importancia suma en el proceso enseñanza aprendizaje: "Más aún, esta situación afecta y es sentida por los estudiantes que no tienen idea de cuál es la naturaleza del problema cuando no han aprendido. En tales situaciones la respuesta del maestro se limita comúnmente a comentarios poco efectivos acerca de: que las calificaciones obtenidas en los exámenes o a decirles que sus trabajos no alcanzaron la calidad esperada, o que el estudiante no se está esforzando lo suficiente". (Domínguez, 2012)

Se conoce que los exámenes son un instrumento más de apoyo para el aprendizaje y no un elemento de evaluación en el más estricto sentido. Los exámenes deben ser un instrumento que retroalimente o redirija el proceso de aprendizaje. Bajo esa perspectiva debe llevarse su construcción.

(Onrubia 2005) refiere las implicaciones que conlleva el aprendizaje en entornos virtuales y de qué se puede hacer desde la enseñanza para promover el aprendizaje, haciendo referencia específicamente a dos de ellas: La primera es la diferencia entre la "estructura lógica" del contenido y la "estructura psicológica".

En la primera muestra una responsabilidad plena del docente para estructurar el objeto de aprendizaje con congruencia, profundidad, pertinencia y dentro del contexto de los planes y programas de estudio, sobre la base de una investigación del estado del arte y de la realización de procesos empíricos metodológicamente realizados, en un ejercicio que va más allá de las aulas y de los tiempos asignados en la administración académica.

En cuanto a la estructura psicológica, es necesario evaluar las estructuras de cada uno de nuestros alumnos, a fin de ajustar los contenidos a sus necesidades de integración y reconstrucción de sus estructuras de conocimiento, apoyados en estrategias de motivación para su realización.

\section{DESCRIPCIÓN DE LA INNOVACIÓN}

El método de aprendizaje basado en la investigación (MABI), nace como una respuesta al interrogante de cómo llevar a las aulas los principios y fundamentos de los Modelos Educativo Institucional y de Integración social, ahí frente al alumno pero también más allá, en los espacios que utiliza para apoyar el aprendizaje. Se establece como elemento fundamental que en este método sea el alumno el centro de la actividad del proceso enseñanza aprendizaje, sin dejar en segundo plano al docente sino buscando una interacción entre ambos en una transformación permanente, dinámica y flexible pero guiados por un elemento integrador como lo es la investigación científica, fuente real y robusta del conocimiento.

El objetivo final de la aplicación de esta estrategia es mejorar y flexibilizar el Método de Aprendizaje Basado en la Investigación (MABI) a fin de que, no perdiendo su esencia, se adapte a las condiciones del docente y de los alumnos de los distintos currículos y niveles, a fin de elevar el nivel de aprendizaje - del proceso y del producto- de los alumnos, mediante la identificación de elementos que se deban integrar o modificar para que el MABI cumpla su cometido con calidad 
Esta se describe en la Planeación didáctica de la Unidad de Aprendizaje en la que se describe el método de aprendizaje basado en la investigación (MABI) que tiene como instrumento natural el uso de las TIC y los alumnos utilizan la web como instrumento de comunicación e información durante el desarrollo de sus diferentes actividades.

\section{PROCESO DE IMPLEMENTACIÓN}

Se consideró para la aplicación del MABI un grupo de alumnos de la 40 Generación de la Carrera de Medicina que cursaron la Unidad Modular: Ciencias Básicas del Aparato Cardiovascular y Sistema Hematopoyético (Bioquímica) del 19 al 22 de abril de 2016, que se muestra en la planeación académica.

\section{INSTITUTO POLITÉCNICO NACIONAL}

\section{CENTRO INTERDISCIPLINARIO DE CIENCIAS DE LA SALUD UNIDAD MILPA ALTA DEPARTAMENTO DE MEDICINA}

\section{Planeación Académica: 40 Gen Medicina}

Competencia: (propósito de la unidad): Integra las funciones y factores que mantienen y modifican la homeostasis con base en los aspectos bioquímicos y fisiológicos del aparato cardiovascular y sistema hematopoyético

\begin{tabular}{|l|l|}
\hline UNIDAD DE APRENDIZAJE & $\begin{array}{l}\text { APARATO CARDIOVASCULAR Y SISTEMA HEMATOPOYETICO, } \\
\text { CIENCIAS BÁSICAS (BIOQUÍMICA) }\end{array}$ \\
\hline DOCENTE: & M en C BLANCA ELISA PÉREZ MAGAÑA \\
\hline
\end{tabular}

CRONOGRAMA: Del 19 al 22 de abril de 2016

\begin{tabular}{|c|c|c|c|c|c|}
\hline $\begin{array}{l}\text { CONTENIDO } \\
\text { TEMÁTICO }\end{array}$ & FЕСНА & $\begin{array}{l}\text { HO- } \\
\text { RAS }\end{array}$ & $\begin{array}{l}\text { ESTRATEGIAS DE } \\
\text { APRENDIZAJE }\end{array}$ & $\begin{array}{l}\text { EVALUACIÓN DE } \\
\text { LOS } \\
\text { APRENDIZAJES }\end{array}$ & $\begin{array}{l}\text { RECURSOS } \\
\text { DIDÁCTICOS }\end{array}$ \\
\hline $\begin{array}{l}\text { Componentes } \\
\text { proteicos de las } \\
\text { células } \\
\text { miocárdicas. } \\
\text {-Organización de } \\
\text { las miofibrillas } \\
\text { ultraestructura de } \\
\text { los miofilamentos. } \\
\text {-Mecanismos de la } \\
\text { contracción } \\
\text { muscular cardiaca } \\
\text {-El corazón como } \\
\text { glándula endocrina. } \\
\text {-Fuentes de energía } \\
\text { para la contracción } \\
\text { muscular: Beta } \\
\text { oxidación, } \\
\end{array}$ & $20 / 04$ & 6 & $\begin{array}{l}\text { Método de aprendizaje } \\
\text { basado en la investigación } \\
\text { (MABI) } \\
\text { Identificación de } \\
\text { conocimiento previo con } \\
\text { lluvia de ideas sobre el } \\
\text { Aparato cardiovascular } \\
\text { El maestro expondrá con } \\
\text { una pequeña introducción } \\
\text { sobre los principales } \\
\text { componentes de la unidad } \\
\text { contráctil del músculo } \\
\text { cardiaco. } \\
\text { Búsqueda selectiva de } \\
\text { información científica } \\
\text { relevante de los contenidos } \\
\text { temáticos en forma }\end{array}$ & $\begin{array}{l}\text { Evaluación de la guía } \\
\text { de discusión No } 1 \text { en } \\
\text { forma individual y el } \\
\text { trabajo grupal que } \\
\text { enviaran al docente a } \\
\text { su correo electrónico }\end{array}$ & $\begin{array}{l}\text { Pizarrón, gis, } \\
\text { borrador. } \\
\text { Retroproyector de } \\
\text { Acetatos } \\
\text { Rortafolios } \\
\text { Video proyector } \\
\text { Material } \\
\text { didáctico: } \\
\text { Presentaciones en } \\
\text { Power Point } \\
\text { Documentos } \\
\text { Informativos del } \\
\text { Ap. Cardiología y } \\
\text { Sistema } \\
\text { Hematopoyético } \\
\text { Evaluaciones }\end{array}$ \\
\hline
\end{tabular}




\begin{tabular}{|c|c|c|c|c|c|}
\hline $\begin{array}{l}\text { Glucolisis aeróbica, } \\
\text { Glucogenolisis y } \\
\text { Sistema } \\
\text { Fosfocreatina. } \\
\text {-Función y } \\
\text { aplicación clínica } \\
\text { de las enzimas } \\
\text { (L.D.H. y C.P.K.). } \\
\text {-Lípidos } \\
\text { Sanguíneos. } \\
\text {-Perfil Bioquímico, } \\
\text {--Patrón } \\
\text { electroforéticos } \\
\text { normal de } \\
\text { lipoproteínas. } \\
\text {-Clasificación de } \\
\text { las } \\
\text { Hiperlipoproteinem } \\
\text { ias Primarias y } \\
\text { Secundaria } \\
\text {-Diagnóstico } \\
\text { clínico y riesgo } \\
\text { coronario en las - } \\
\text { Hiperlipoproteinem } \\
\text { ias. } \\
\text {-El colesterol en la } \\
\text { patología vascular. } \\
\text {-Hierro; digestión y } \\
\text { mecanismo de } \\
\text { absorción } \\
\text { intestinal, } \\
\text { transporte extra e } \\
\text { intracelular del } \\
\text { hierro, factores que } \\
\text { intervienen en la } \\
\text { absorción del } \\
\text { hierro } \\
\text {-Hemoglobina: } \\
\text { composición } \\
\text { química y síntesis } \\
\text { de la hemoglobina, } \\
\text {-Importancia de la } \\
\text { vitamina C, B12 y } \\
\text { síntesis de la } \\
\text { hemoglobina }\end{array}$ & $22 / 04$ & 6 & $\begin{array}{l}\text { individual para la } \\
\text { resolución de las guías de } \\
\text { discusión } 1,2 \text { y } 3 \\
\text { Discusión la guía de } \\
\text { discusión } 1 \text { en forma } \\
\text { individual y grupal para, } \\
\text { exponer en una plenaria el } \\
\text { análisis del mismo } \\
\text { Discusión la guía de } \\
\text { discusión } 2 \text { en forma } \\
\text { individual y grupal para, } \\
\text { exponer en una plenaria el } \\
\text { análisis del mismo } \\
\text { El maestro expondrá y dará } \\
\text { a conocer la importancia del } \\
\text { sistema Hematopoyético } \\
\text { Discusión } \\
\text { la guía de discusión } 3 \text { en } \\
\text { forma } \\
\text { individual y grupal y } \\
\text { exponer en una plenaria el } \\
\text { análisis del mismo }\end{array}$ & $\begin{array}{l}\text { Evaluación la guía de } \\
\text { discusión No } 2 \text { en } \\
\text { forma individual y el } \\
\text { trabajo grupal que } \\
\text { enviaran al docente a } \\
\text { su correo electrónico } \\
\\
\text { Evaluación la guía de } \\
\text { discusión No } 3 \text { en } \\
\text { forma individual y el } \\
\text { trabajo grupal que } \\
\text { enviaran al docente a } \\
\text { su correo electrónico } \\
\text { Portafolio de } \\
\text { evidencias: } \mathbf{5 0 \%} \\
\text { (actividades } \\
\text { individuales y } \\
\text { grupales) + 50 \% } \\
\text { Evaluación sumativa }\end{array}$ & $\begin{array}{l}\text { formativas que se } \\
\text { frecen en la } \\
\text { WEB mediante la } \\
\text { memoria virtual } \\
\text { www.dropbox.com } \\
\text { O bien en el correo } \\
\text { electrónico de la } \\
\text { generación }\end{array}$ \\
\hline Total de horas & & 24 & & & \\
\hline
\end{tabular}

\section{EVALUACIÓN DE LOS RESULTADOS}

La evaluación total que se consideró para aprobar el curso fue, la suma del 50\% del portafolio de evidencias con la búsqueda selectiva de información científica relevante de las guías de discusión 1, 2 y 3 en forma individual. Presentación de trabajos grupales sobre la base de la integración de trabajos individuales. Lecturas y análisis grupales sobre la base de sus referencias bibliográficas. Se maneja el concepto de participación referenciada, que consiste en participar en el desarrollo de la Unidad de 
aprendizaje únicamente cuando se cuenta con una referencia bibliográfica o de investigación. Este fue el elemento de control y seguimiento del aprendizaje, utilizando como evidencias los trabajos enviados vía internet y guardados en una carpeta por alumno y el otro 50\% fue la evaluación sumativa representado por el examen. Finalmente, la calificación final se basó en la evaluación del proceso de aprendizaje y el aprendizaje mismo.

Se utilizaron las TICS como instrumento de apoyo y para no utilizar únicamente las cuatro paredes del salón en el proceso de aprendizaje. Con la diferencia de que se brindó asesoría correo electrónico para envío de trabajos; habiéndose revisado los trabajos por esta vía, se les retroalimentaba de la misma manera.

Al grupo se les proporcionó el material básico y complementario suficiente mediante una página en internet, utilizando la memoria virtual www.dropbox.com

El control de las actividades se llevó con el apoyo de una lista de asistencia y una lista de cotejo puesta a la vista del grupo.

De la aplicación del (MABI)

Se realizó una encuesta escrita de 5 preguntas.

Preguntas de evaluación oral del MABI a los alumnos que llevaron el curso

1. ¿Qué dificultades y facilidades tuvieron para aplicar el MABI, docentes y alumnos?

2. ¿Consideran que el MABI favoreció el aprendizaje aprovechamiento y de sus alumnos?

3. ¿Existe un elemento que evidencie la mejoría en el proceso de aprendizaje? Sí, sí, cuál y si, no por qué?

4. ¿Consideran que el MABI facilita la evaluación de aprovechamiento?

5. ¿Qué modificarías para mejorar el MABI?

Los comentarios en forma escrita de los alumnos sobre el Método Basado en la Investigación fuero los siguientes:

\subsection{DIFICULTADES}

- Las fuentes de información no son $100 \%$ confiables o están incompletas.

- Las páginas con respaldo científico (revistas o artículos) no son gratuitas

- El costo de los libros es alto

- Los libros de la biblioteca son insuficientes para la cantidad de alumnos

\subsection{VENTAJAS}

- Se contaba con las herramientas necesarias, tales como computadora, acceso a internet.

- Se puede trabajar desde su casa, en el horario más conveniente y al llegar a clase comparar, discutir y compartir la información, lo cual reafirma conocimientos y también se compara y se comparten las fuentes bibliográficas

- Favorece el aprendizaje y dominio sobre los temas ya que fue necesario leer, entender todo antes de la clase y durante la clase solo se homogenizan los conocimientos y se aclaran dudas, lo cual se refleja positivamente en él logró de los objetivos del programa.

- $\mathrm{Al}$ investigar por si mismo los temas se van asimilando y dominando poco a poco, los temas contenidos en el examen

- El elemento que demuestra que este método es útil son los resultados del examen, ya que cuando se realiza este, ya se cuenta con los recursos para obtener resultados satisfactorios y resolverlo fácil y correctamente 
- Se disminuye la dificultad para evaluar al alumno solo con el examen, ya que se considera además de este lo recopilado en el portafolio de evidencias

- Al realzar la investigación bibliográfica se evalúa también el aprovechamiento al fomentar la participación del alumno durante la clase

- Se adquieren habilidades para investigar y realizar trabajos de investigación de calidad

- No solo se quedan los conocimientos adquiridos, se estimula en análisis critico

\subsection{SUGERENCIAS DE MEJORA DE LA ESTRATEGIA}

- Que la escuela tenga un Blog o Plataforma en el cual se puedan descargar libros digitales y así ahorrar tiempo en la búsqueda de información

- Proponer páginas web confiables en este caso de Bioquímica del Aparato Cardiovascular y Sistema Hematopoyético

\section{CONCLUSIONES}

De acuerdo a mi experiencia docente en el CICS-UMA es que el índice de reprobación de los alumnos ha bajado. Ya que el número de alumnos reprobados eran entre 10 o 12 alumnos por grupo en las generaciones pasadas en que solo se aplicaba un examen único y ahora después de aplicar este método el índice de reprobación es entre 2 y 4 alumnos reprobados por grupo de un total de 70 alumnos. El aprovechamiento de los alumnos fue superior lo cual se refleja en el índice de reprobación fue mínimo.

Por lo que considero muy importante considerar este método basado en la investigación, que implica más trabajo para el docente sí y para los alumnos también, pero con la suma de las actividades que envían los alumnos, considero que los vamos conociendo más y se van retroalimentando los conocimientos día a día y la evaluación final no solo se basa en el examen. Este método se adapta a las condiciones del docente y de los alumnos, a fin de elevar el nivel de aprendizaje de los alumnos, mediante la identificación de elementos que se deban integrar o modificar para que el MABI cumpla su cometido con calidad. 


\section{REFERENCIAS}

Domínguez N. J. A. y Col. (2012). Competencias Docentes para la innovación y la investigación educativa. Comparativa entre el aprendizaje basado en proyectos y el aprendizaje basado en problemas. Centro de Formación e Innovación Educativa. IPN. México.

Light G. y Roy C. (2009), Learning and teaching in higher education: the reflective professional, Second Edition, Editorial Paul Chapman Publishing. Great Britain.

Gregorio E. P. (2007). El docente-investigador: Un mapa para explorar un territorio complejo. Ed. Primera. Editorial Laboratorio de Alternativas Educativas. Facultad de Ciencias Humanas. Universidad Nacional de San Luís. Argentina.

Onrubia, J. (2005). Aprender y enseñar en entornos virtuales: actividad conjunta, ayuda pedagógica y construcción del conocimiento. RED. Revista de Educación a Distancia, número monográfico II.

Un Nuevo Modelo Educativo para el IPN. Materiales para la reforma. Primera edición: (2004). Instituto Politécnico Nacional. Pág.9-11. 